\title{
Preliminary Multi-Variable Cost Model for Space Telescopes
}

\author{
H. Philip Stahl ${ }^{\mathrm{a}}$ and Todd Henrichs ${ }^{\mathrm{b}}$ \\ ${ }^{a}$ NASA MSFC, Huntsville, AL 35821; and \\ ${ }^{\mathrm{b}}$ Department of Mathematical Sciences, Middle Tennessee State University
}

\begin{abstract}
Parametric cost models are routinely used to plan missions, compare concepts and justify technology investments. This paper reviews the methodology used to develop space telescope cost models; summarizes recently published single variable models; and presents preliminary results for two and three variable cost models. Some of the findings are that increasing mass reduces cost; it costs less per square meter of collecting aperture to build a large telescope than a small telescope; and technology development as a function of time reduces cost at the rate of $50 \%$ per 17 years.
\end{abstract}

Keywords: Space Telescope Cost Model, Parametric Cost Model

\section{INTRODUCTION}

Multivariable parametric cost models for space telescopes provide several benefits to designers and space system project managers. They identify major architectural cost drivers and allow high-level design trades. They enable cost-benefit analysis for technology development investment. And, they provide a basis for estimating total project cost. A survey of historical models revealed that there is no definitive space telescope cost model. In fact, the published models vary greatly. [1] Therefore, the opportunity exists to develop a multi-variable parametric cost model for space telescopes that encompasses the latest available data and applies rigorous analytical techniques. The first step in this process was to develop a single variable parametric cost model for space telescopes. [2]

Cost and engineering data has been collected on 59 different parameters for 23 different UV, optical or infrared space telescopes. (Table 1 and Table 2)

\begin{tabular}{|c|c|}
\hline \multicolumn{2}{|c|}{ Table 1: UV/OIR Cost Model Missions Database } \\
\hline UV/Optical Telescopes & Infrared Telescopes \\
\hline EUVE & CALIPSO \\
FUSE & Herschel \\
GALEX & ICESat \\
HiRISE & IRAS \\
HST & ISO \\
HUT & JWST \\
IUE & SOFIA \\
Kepler & Spitzer (SIRTF) \\
Copernicus (OAO-3) & TRACE \\
SOHO/EIT & WIRE \\
UIT & WISE \\
WUPPE & \\
\hline
\end{tabular}

Statistical correlations have been evaluated between 19 of 59 variables. And, these parameters have been used to develop single and multi-variable cost estimating relationships (CERs) which are evaluated for their 'goodness'. For the purpose of this paper, Optical Telescope Assembly (OTA) is defined as the space observatory subsystem which collects electromagnetic radiation and focuses it (focal) or concentrates it (afocal). An OTA consists of the primary mirror, secondary mirror, auxiliary optics and support structure (such as

\begin{tabular}{|c|c|}
\hline \multicolumn{2}{|c|}{$\begin{array}{l}\text { Table 2: Cost Model Variables Study } \\
\text { and the completeness of data knowledge }\end{array}$} \\
\hline Parameters & $\%$ of Data \\
\hline OTA Cost & $89 \%$ \\
\hline Total Phase A-D Cost w/o LV & $84 \%$ \\
\hline Aperture Diameter & $100 \%$ \\
\hline Avg. Input Power & $95 \%$ \\
\hline Total Mass & $89 \%$ \\
\hline OTA Mass & $89 \%$ \\
\hline Spectral Range & $100 \%$ \\
\hline Wavelength Diffraction Limit & $63 \%$ \\
\hline Primary Mirror Focal Length & $79 \%$ \\
\hline Design Life & $100 \%$ \\
\hline Data Rate & $74 \%$ \\
\hline Launch Date & $100 \%$ \\
\hline Year of Development & $95 \%$ \\
\hline Technology Readiness Level & $47 \%$ \\
\hline Operating Temperature & $95 \%$ \\
\hline Field of View & $79 \%$ \\
\hline Pointing Accuracy & $95 \%$ \\
\hline Orbit & $89 \%$ \\
\hline Development Period & $95 \%$ \\
\hline Average & $88 \%$ \\
\hline
\end{tabular}
optical bench or truss structure, primary support structure, secondary support structure or spiders, etc.). An OTA does not include science instruments or spacecraft subsystems. And, cost is defined as prime contract cost without any NASA labor or overhead. Total mission cost is defined as Phase A-D cost, excluding: launch cost; costs associated with NASA labor (civil servant or support contractors) for program management, technical insight/oversight; or any NASA provided ground support equipment, e.g. test facilities. Accounting for NASA overheads would increase the cost by at least $10 \%$ and maybe as much as $33 \%$. 
Four single variable cost estimating relationships (CERs) have been developed. These CERs estimate OTA cost and total mission cost as a function of OTA diameter, OTA mass and total mission mass. [2] This paper reviews those results including the finding that 'attached' OTAs with mass 10X larger than 'free-flying' OTAs are $60 \%$ less expensive; tests the historical Horak model against the data base; and presents preliminary results regarding the development of a multivariable cost model.

\section{MODEL CREATION}

The first step in creating a statistical cost model is to start with the Cross Correlation Matrix (Figure 1) and look for variables which are highly correlated with cost. When using a cross-correlation matrix, there are several things to consider. First, the higher the correlation value, the greater the cost variation explained by that variable. Second, the sign of correlation is important. It must be consistent with known engineering design principals and manufacturing processes. Third, for multi-variable models, we want variables which independently effect cost. Variables which 'crosstalk' with each other are multicollinear.

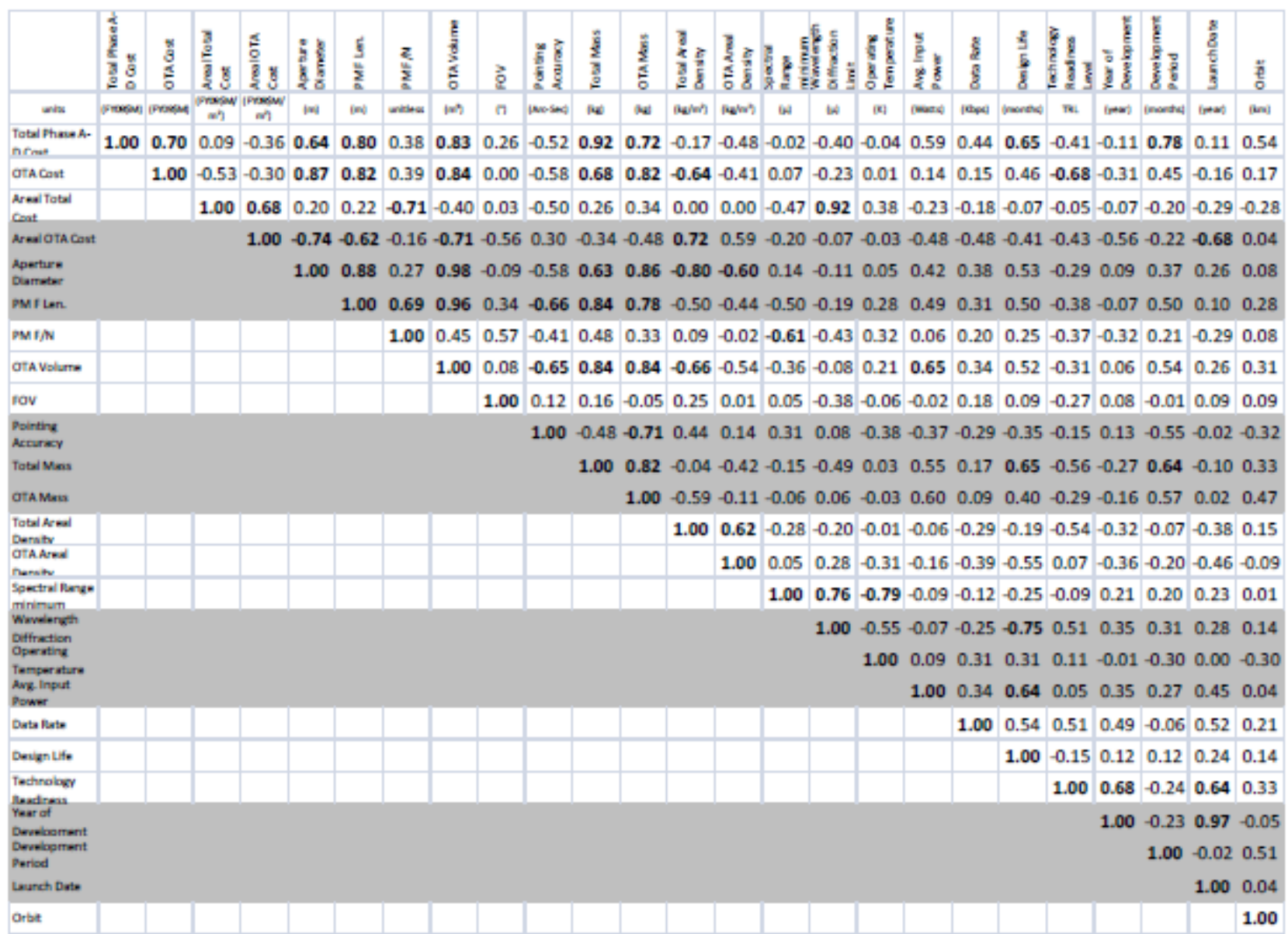

Figure 1: Cross-Correlation Matrix of data base for 19 Free-Flying Space Telescope Systems. Correlations which are at least $95 \%$ significant are Bolded, e.g. for 12 data points a correlation of greater than $60 \%$ is significant to better than $95 \%$.

The second step is to understand the statistical indicators of 'Goodness of Fit' and 'Significance'. Goodness of Fit is tested via a range of statistical measures, including Pearson's $\mathrm{r}^{2}$ coefficient, Student T-Test $p$-value and standard percent error (SPE). Pearson's $r^{2}$ (typically denoted as just $r^{2}$ ) describes the percentage of agreement between the model and the actual cost. For multi-variable models, we use Adjusted Pearson's $r^{2}$ (or $r^{2}$ adj $)$ which accounts for the number of data points and the number of variables. In general, the closer $\mathrm{r}^{2}\left(\right.$ or $\mathrm{r}^{2}$ adj) is to 1.0 or $100 \%$, the better the model. SPE is a normalized standard deviation of the fit residual (difference between data and fit) to the fit. The closer SPE is to 0, the better the fit. Please note that since SPE is normalized, a small variation divided by a very small parameter coefficient can yield a very large SPE. The p-value is the probability that a fit or correlation would occur if the variables are 
independent of each other. The closer the p-value is to 0 , the more significant the fit or correlation. The closer it is to 1 , the less significant. If the p-value for a given variable is small, then removing it from the model would cause a large change to the model. If it is large, then removing the variable will have a negligible effect. Also, it is important to consider how many data points are included in a given correlation, fit or regression.

Given the complexity and data density of Figure 1, Table 3 digests the cross-correlation between specific key parameters and Total Mission Cost, OTA Cost and OTA Areal Cost (where areal cost is defined at OTA cost divided by OTA collecting area). For each parameter, Table 3 reports its $r^{2}$ correlation to cost, the correlation's p-value and the number of data points in the correlation. Diameter appears to be the most significant cost driver. So, in addition to total cost and OTA cost we have examined OTA Areal Cost, i.e. OTA Cost per unit Area of Primary Mirror collecting aperture.

\begin{tabular}{|l|l|l|l|l|l|l|l|l|l|}
\hline \multicolumn{1}{|c|}{ Table 3: Cross-Correlation Results of Specific Parameters vs Cost } \\
\hline \multirow{3}{*}{ Parameter } & \multicolumn{3}{c|}{ Total Cost } & \multicolumn{3}{c|}{ OTA Cost } & \multicolumn{3}{c|}{ OTA Areal Cost } \\
\cline { 2 - 12 } & Corr & $\mathrm{p}$ & $\mathrm{N}$ & Corr & $\mathrm{p}$ & $\mathrm{N}$ & Corr & $\mathrm{p}$ & $\mathrm{N}$ \\
\hline Diameter & .68 & .007 & 14 & .87 & 0 & 16 & -.71 & .005 & 14 \\
\hline Focal Length & .82 & .002 & 11 & .82 & .001 & 12 & -.42 & .194 & 11 \\
\hline Pointing Accuracy & -.53 & .061 & 14 & -.64 & .011 & 15 & .47 & .087 & 14 \\
\hline Total Mass & .92 & 0 & 15 & .68 & .005 & 15 & -0 & .997 & 15 \\
\hline OTA Mass & .72 & .002 & 15 & .82 & 0 & 15 & -.47 & .074 & 15 \\
\hline Spectral Min & -.02 & .934 & 16 & .07 & .804 & 17 & -.23 & .383 & 16 \\
\hline Operating Temp & -.04 & .884 & 16 & 0 & .975 & 16 & -.07 & .802 & 16 \\
\hline Electrical Power & .59 & .021 & 15 & .14 & .611 & 16 & -.05 & .862 & 16 \\
\hline Design Life & .65 & .007 & 16 & .46 & .064 & 17 & -.20 & .454 & 16 \\
\hline TRL & -.41 & .307 & 8 & -.68 & .061 & 8 & -.29 & .481 & 8 \\
\hline Development Period & .78 & .001 & 15 & .45 & .083 & 15 & .14 & .830 & 15 \\
\hline Launch Year & .11 & .675 & 16 & -.16 & .533 & 17 & -.34 & .204 & 16 \\
\hline
\end{tabular}

Diameter is correlated with all three with a significance of greater than $99 \%$. Primary Mirror Focal Length is also a significant correlation, but as we will discover later, it is multi-collinear with Diameter. The assumed explanation is that all space telescopes tend to have the same basic PM F/\#. Pointing Accuracy has reasonable correlation with cost. And, as expected from engineering judgment, it has significant correlation (99\% confidence level) with diameter and OTA mass. Interesting, as will be discussed later, pointing is not multicollinear with either. As expected, Total Mass correlates most significantly with Total Cost while OTA Mass correlates most significantly with OTA Cost. Unexpectedly, Minimum Spectral Range Value and Operating Temperature do not have a significant correlation with any Cost. However, as we will show later, Spectral Minimum does have a role in multi-variable cost models. As expected Electrical Power, Design Life and Development Period have significant correlations (99\% confidence) with Total Cost. Also unexpected is that TRL and Launch Year do not have significant correlations. But, as we will discuss later, they both have roles in multi-variable cost models. One problem with TRL is that there are only 8 data points. Also, it is a qualitative and not a quantitative parameter.

\section{SUMMARY OF SINGLE VARIABLE COST MODEL RESULTS}

Four single variable cost estimating relationships (CERs) have been developed for OTA cost and total mission cost as a function of OTA diameter, OTA mass and total mission mass. [2] These models were developed with and without JWST. The benefit of including JWST is that it is the most current mission. The disadvantage is that its cost is not yet final. For the purpose of this paper, we will include the 2009 JWST C/D final cost estimate. In general, including JWST does affect the model $r^{2}$ adj but does not increase the noisiness of the fit as represented by the SPE. Additionally, these models are developed only for free-flying missions. Of the 23 missions in the data base, there are 19 free flying telescopes (17 for which we have OTA cost data) and 4 that are attached ( 3 to the Space Shuttle Orbiter and SOFIA to a Boeing 747 airplane). As will be discussed below with regard to mass models, attached missions have a significantly different cost dependency than free-flying missions. Therefore, we excluded attached missions from the models.

Figure 3 plots OTA Cost for free-flying space telescopes as a function of Primary Mirror Diameter. The regression fit for this data is:

\section{OTA Cost $~$ Aperture Diameter ${ }^{1.2}$}

$$
\left(\mathrm{N}=17 ; \mathrm{r}^{2}=75 \% ; \mathrm{SPE}=79 \%\right) \text { with } 2009 \mathrm{JWST}
$$

Note that the Chandra data point is included only for reference. It is not included in the regression. And, it is inserted based upon the equivalent normal incidence mirror diameter it would have if all of its x-ray mirrors were unrolled.

Given that the OTA cost might be dominated by the large apertures for HST and JWST, a model was also created for normalized Areal OTA Cost (Figure 4):

$$
\text { OTA Areal Cost } \sim \text { Aperture Diameter }{ }^{-0.74} \quad\left(N=17 ; r^{2}=55 \% \text {; SPE }=78 \%\right) \text { with JWST }
$$


A key finding of this analysis is that Areal Cost decreases with aperture size. It is less expensive per photon to build a large aperture telescope than a small aperture telescopes. Large aperture telescopes provide a better ROI.

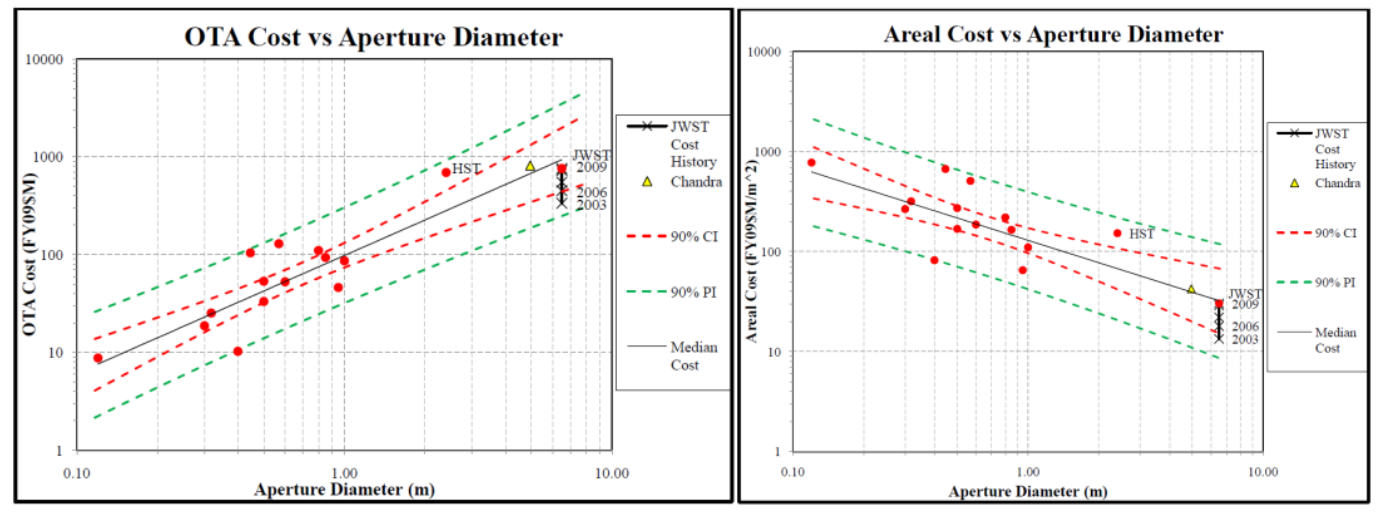

Figure 3: OTA Cost vs Aperture Diameter scaling law for 17 free flying UV/OIR systems (including 2009 JWST). Plot includes $90 \%$ confidence and prediction intervals, and data points. Chandra data

Figure 4: OTA Areal Cost vs Aperture Diameter scaling law for 17 free flying UV/OIR systems (including 2009 JWST). Plot includes $90 \%$ confidence and prediction intervals, and data points. point is not included in the regression.

Chandra data point is not included in the regression.

From both an engineering and a science perspective, aperture diameter is the best parameter upon which to build a space telescope cost model. Aperture defines the observatory's science performance and determines the payload's size and mass. And, while the results are consistent with some historical cost models, our results invalidate long held 'intuitions' which are often purported to be 'common knowledge'. Space telescope costs vary almost linearly with diameter and not to a power of $1.6 \mathrm{X}$ or $2.0 \mathrm{X}$ or even $2.8 \mathrm{X}$. But, a model based on diameter alone has only an $\sim 80 \%$ agreement with the OTA cost data and $~ 55 \%$ agreement with the OTA areal data. Therefore, other factors must influence cost. The next step is to develop a multi-variable cost model using multi-variable regression techniques.

While aperture diameter is the single most important parameter driving science performance, total system mass determines what vehicle can be used to launch. Significant engineering costs are expended to keep a given payload inside of its allocated mass budget. This includes light-weighting mirrors and structure. Therefore, mass is a potential CER which requires study. Figure 5 plots Total Cost for Free-Flying Missions vs Total Mission Mass. The regression of this data is:

\section{Total Cost $\sim$ Total Mass ${ }^{1.12}\left(\mathrm{~N}=15 ; \mathrm{r}^{2}=86 \% ;\right.$ SPE $\left.=71 \%\right)$ with JWST}

Figure 6 plots OTA Cost vs OTA Mass for both free-flying and attached missions. The regression for only the freeflying missions is:

\section{OTA Cost $\sim$ OTA Mass ${ }^{0.72}\left(\mathrm{~N}=15 ; \mathrm{r}^{2}=92 \% ;\right.$ SPE $\left.=93 \%\right)$ with JWST}

While OTA Mass may appear to be a good indicator of OTA Cost because it has the highest Pearson's $\mathrm{r}^{2}$, it also has the highest SPE. In general mass should be avoided as a CER because it is a secondary indicator. Mass depends upon the size of the telescope. Bigger telescopes have more mass. And, bigger telescopes typically require bigger spacecraft and bigger science instruments which both require more power - all which require more mass. And, because many missions are designed to a mass-budget defined by launch vehicle constraints, the result can be a very complex, risky, and expensive mission architecture when trying to extend the state-of-the-art in either wavelength or aperture. An indication of this is given in Figure 5 where JWST has nearly half the total mass of HST but still has a higher total mission cost because JWST is much more complex than HST. But, this does not have to be the case. The key finding of Figure 6 (as indicated by the square data points) is that attached OTAs are $\sim 10 \mathrm{X}$ more massive and $\sim 60 \%$ less expensive than freeflying missions. This finding actually invalidates the 'common assumption' that the more massive the mission the more expensive the mission. The only reason that more massive missions are more expensive is because they have more 'stuff'. When one compares missions with similar performance properties, it is less expensive to design, build and fly a simple mission with more mass than a lightweight complex mission. Therefore, maybe the best way to reduce the cost 
of future large aperture space telescopes is to develop cost effective heavy lift launch vehicles which will enable mission planners to trade complexity for mass.

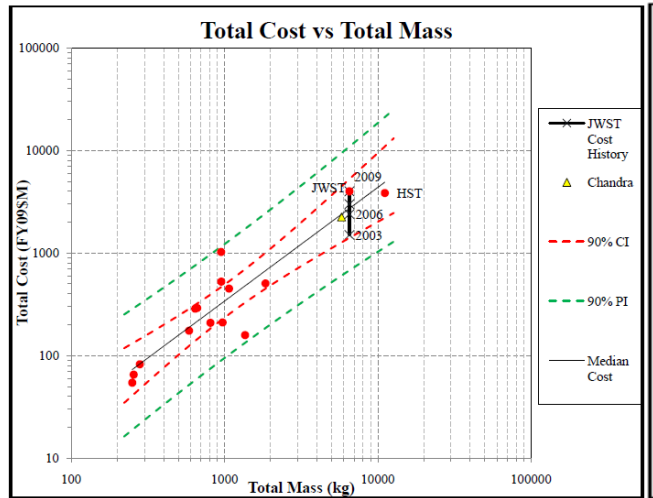

Figure 5: Total Cost vs Total Mass scaling law for free-flying UV/OIR space telescopes (including 2009 JWST). Plot includes 90\% confidence and prediction intervals, and data points. Chandra data point is not included in the regression.

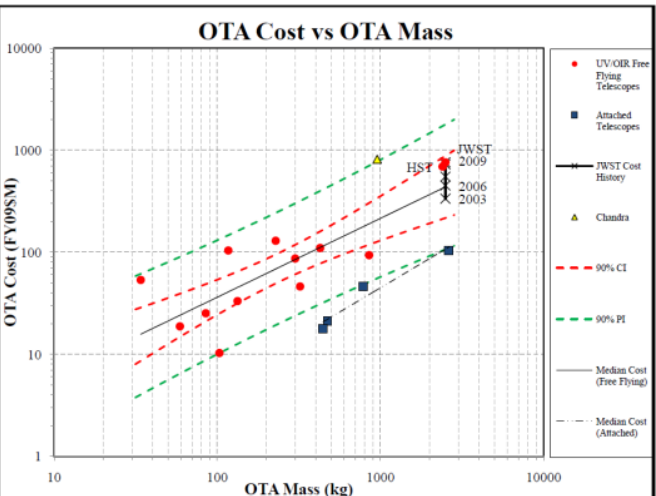

Figure 6: OTA Cost vs OTA Mass scaling law for free-flying UV/OIR space telescopes (including 2009 JWST). Plot includes 90\% confidence and prediction intervals, and data points. Chandra data point is not included in the regression.

\section{TEST OF HISTORICAL MODELS}

One of the goals of the study was to test historical models against our data base. Of all the historical models, the Horak model is the easiest to test. Our database has parameters equivalent to the Horak database. And, Horak published the details of his statistical fit including his Student-T Test Confidence values (Figure 7):

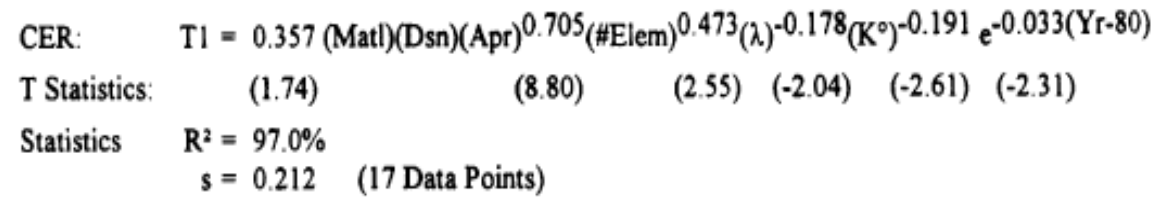

Figure 7: Horak Cost Estimating Relationship with Statistical Details

For this comparison, we ignore the Material (glass vs metal) and Design (on vs off-axis) factors and concentrate on the parameters with power terms. The first step is to convert the Horak T Statistics for each parameter into p-values:

$\begin{array}{lccccc}\text { Parameter } & \text { Apr } & \text { \#Elem } & \text { Wave } & \text { Temp } & \text { Year } \\ \text { T Statistics: } & 8.80 & 2.55 & -2.04 & -2.61 & -2.31 \\ \text { p-values: } & 0.00 & 0.022 & 0.059 & 0.020 & 0.036\end{array}$

Based on 17 data points, the reported $\mathrm{T}$ statistics and p-values indicate that all variables in Horak's model are significant. And, given that he reported an $\mathrm{R}^{2}=97 \%$, his model yielded a good fit to his data. Next, we regress the Horak parameters against our data base yielding:

\begin{tabular}{|c|c|c|c|c|c|}
\hline arameter & Apr & \#Elem & Wave & Temp & Year \\
\hline istics: & 9.34 & -1.03 & -0.22 & -0.38 & -2.80 \\
\hline p-values: & 0.00 & 0.320 & 0.829 & 0.710 & 0.014 \\
\hline
\end{tabular}

For our data, based on 16 data points, only Diameter and Launch Year are significant and the fit has a good $\mathrm{R}^{2}=90.8 \%$ and $\mathrm{R}_{\text {adj }}^{2}=86.2 \%$. (Please note: $\mathrm{R}^{2}$ statistics, which are calculated in log-space, are reported so as to match Horak's measures of 'goodness'.) These T statistics and p-values are generated from the t-test for the coefficients which tests whether the coefficient is equal to zero or not. The p-value represents the probability of getting the result we got (the coefficient) if the actual coefficient equals zero. The standard is to reject the coefficient (argue that it is really zero) if 
the p-value is greater than 0.05 (corresponding to a 5\% chance of getting the coefficient we got if it is really zero). For this model regression against our data base, we cannot claim that the exponents for \#Elem, $\lambda$, and $\mathrm{K}^{\circ}$ are statistically different than zero. Thus those terms have little effect on the model and are not drivers of telescope cost. Removing those variables yields the following model:

$\begin{array}{lcc} & \text { OTACost } \sim \text { Diam }^{1.33} e^{-0.0434(Y r-80)} \\ \text { T Statistics: } & 10.61 & -4.22 \\ \text { p-values: } & 0.00 & 0.001\end{array}$

Based on 17 data points, both Diameter and Launch Year are significant and the fit has a good $\mathrm{R}^{2}=89.2 \%$ and $\mathrm{R}_{\text {adj }}^{2}=87.6 \%$. The $\mathrm{R}^{2}$ is marginally smaller after removing the three variables and the $\mathrm{R}_{\text {adj }}^{2}$ increases from the removal.

The explanation is in the databases. The Horak data base consists mostly of DoD strategic systems most of which were laboratory experiments that were never deployed. Of the systems which were flown, most were airframe or missile systems. Our database consists entirely of space telescope missions.

\section{MULTI-VARIABLE PARAMETRIC MODEL}

The first step in developing a multi-variable parametric model is to start with a single variable model and evaluate its statistics 'goodness'. For OTA Cost, we start with Aperture Diameter. Then we perform a two variable regression of Diameter and each of the other parameters under study - one parameter at a time - and evaluate the statistical 'goodness' of each regression. Once a good two variable model is selected, the process is repeated to add a third variable. This process is generally called step-wise regression.

There are five specific criteria that must be satisfied when adding parameters to a multi-variable model:

The correlation of each variable must be 'significant'. An arbitrary criterion is that the parameter's p-value must be less than 0.10 (for a $90 \%$ confidence limit) but in some cases, we will consider variables whose correlations are significant only at the $80 \%$ confidence level.

The parameter's coefficient must be 'consistent' with engineering judgment. For example, it violates engineering judgment if the coefficient for TRL is positive. This would indicate that the higher the TRL level the higher the cost of a telescope or mission.

The addition of a variable should 'increase' Pearson Adjusted $r^{2}$. The close $r^{2}$ adj is to 1.0 the better the model agrees with the data.

The addition of a variable should 'decrease' SPE. The close SPE is to 0.0 the less noise there is in the fit.

The parameters should not be multicollinear. However, this rule may be violated with proper justification. Although at this point we have not identified such a justification.

\subsection{Two-Variable Models}

Figure 8 summarizes the results of a two variable model regression for OTA Cost as a function of Aperture Diameter and a second variable. Three parameters have significance greater than 98\%: TRL, Year of Development (YoD) and Launch Year (LYr). The Diameter + TRL model has a slightly higher $r_{\text {adj }}^{2}$ than the other models, but it also has a high SPE. This may be because of the relatively few TRL data points in our data base. Or, it may be because TRL value is subjective and thus has a natural 'fuzziness' to its data values. Based on coefficient significance, other parameters of potential interest are Field of View (82\%), OTA Mass (74\%), OTA Areal Density (74\%), Power (77\%) and Data Rate $(72 \%)$. But, all, except Data Rate, do not simultaneously increase $r^{2}$ adj and decrease SPE. And, some, such as FOV, are particularly poor. It should also be noted that OTA Mass is multicollinear with Aperture Diameter - which only makes sense, i.e. the larger the telescope, the more mass it should have. Therefore, mass is not a good second variable candidate. For the purpose of future three variable model regressions, we will use YoD or LYr.

Both YoD and LYr have similarly high $\mathrm{r}_{\text {adj }}^{2}$ values and significantly lower SPE values. And, if you round significant digits, each model is virtually identical:

$$
\begin{array}{ll}
\text { OTA Cost } \sim \mathrm{D}^{1.34} \mathrm{e}^{-0.04(\mathrm{LYr}-1960))} & \left(N=17, r_{a d j}^{2}=93 \% ; S P E=39 \%\right) \\
\text { OTA Cost } \sim \mathrm{D}^{1.27} \mathrm{e}^{-0.04(\mathrm{YoD}-1960))} & \left(N=16, r_{a d j}^{2}=95 \% ; S P E=39 \%\right)
\end{array}
$$


At this stage of our study, we have not determined which parameter to use. Launch Year has the advantage that it is a definite date, but it also has the disadvantage that a launch can be delayed. And, while a launch delay tends to increase the total mission cost, it may or may not increase the OTA cost. Year of Development yields a slightly better regression, but its exact date is subject to definition. Is it the Start of Phase A or B or C? Regardless, the message of either 'year' model is clear: technology improvements reduce OTA cost as a function of time by approximately $50 \%$ every 17 years. Further cost reductions could probably be obtained if a procurement strategy was pursued to maintain an industrial infrastructure and a skilled workforce by making multiple system procurements spread out over many years.

\begin{tabular}{|c|c|c|c|c|c|c|c|c|c|c|c|c|c|c|c|c|c|c|}
\hline & coef & $\mathrm{p}$ & \multicolumn{16}{|c|}{ OTA Cost versus Diameter and V2 } \\
\hline 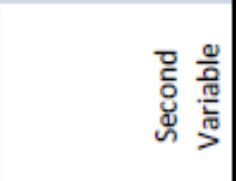 & 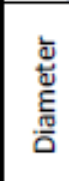 & $\frac{\stackrel{9}{ㅇ ㅡ ㅁ ~}}{\pi}$ & $\begin{array}{l}\dot{c} \\
\stackrel{9}{a} \\
\sum_{a}^{u}\end{array}$ & \multicolumn{2}{|c|}{$\sum_{\substack{L \\
a}}^{z}$} & $\begin{array}{l}\stackrel{0}{E} \\
\frac{D}{D} \\
\frac{5}{5}\end{array}$ & 己े & \multicolumn{2}{|c|}{ 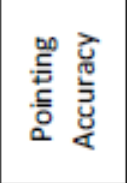 } & \multicolumn{2}{|c|}{ 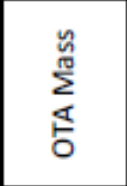 } & \multicolumn{2}{|c|}{ 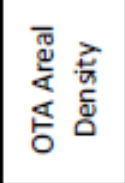 } & \multicolumn{2}{|c|}{ 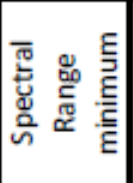 } & 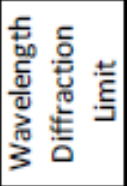 & \multicolumn{2}{|c|}{ 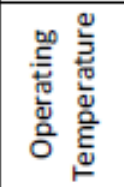 } \\
\hline Diameter & 1.20 & 0.00 & \begin{tabular}{|l|l|}
0.68 & 0.27 \\
\end{tabular} & 1.05 & 0.00 & \begin{tabular}{|l|l|}
-0.02 & 0.99 \\
\end{tabular} & \begin{tabular}{|l|l|}
1.16 & 0.01 \\
\end{tabular} & 1.14 & 0.00 & 0.76 & 0.12 & 1.45 & 0.00 & 1.22 & 0.00 & \begin{tabular}{|l|l|}
1.19 & 0.00 \\
\end{tabular} & 1.21 & 0.00 \\
\hline Second Variable & - & - & \begin{tabular}{|l|l|}
0.35 & 0.45 \\
\end{tabular} & 0.26 & 0.57 & \begin{tabular}{|l|l|}
0.35 & 0.45 \\
\end{tabular} & \begin{tabular}{|l|l|}
-0.26 & 0.18 \\
\end{tabular} & -0.05 & 0.45 & 0.35 & 0.26 & 0.35 & 0.26 & -0.04 & 0.63 & \begin{tabular}{|l|l|}
-0.10 & 0.55 \\
\end{tabular} & -0.01 & 0.96 \\
\hline Adjusted r2 & \multicolumn{2}{|c|}{$73 \%$} & $71 \%$ & \multicolumn{2}{|c|}{$71 \%$} & $71 \%$ & $14 \%$ & \multicolumn{2}{|c|}{$73 \%$} & \multicolumn{2}{|c|}{$83 \%$} & \multicolumn{2}{|c|}{$83 \%$} & \multicolumn{2}{|c|}{$73 \%$} & $75 \%$ & \multicolumn{2}{|c|}{$71 \%$} \\
\hline SPE & \multicolumn{2}{|c|}{$79 \%$} & $77 \%$ & \multicolumn{2}{|c|}{$78 \%$} & $77 \%$ & $73 \%$ & \multicolumn{2}{|c|}{$78 \%$} & \multicolumn{2}{|c|}{$83 \%$} & \multicolumn{2}{|c|}{$83 \%$} & \multicolumn{2}{|c|}{$84 \%$} & $95 \%$ & 82 & $\%$ \\
\hline$n$ & 1 & 7 & 13 & 1 & 3 & 13 & 13 & 16 & & 1 & 5 & 15 & & 1 & 7 & 11 & 1 & 6 \\
\hline Multicollinearity? & $\mathrm{N} /$ & $A$ & Yes & $\mathrm{N}$ & 0 & Yes & No & $\mathrm{Nc}$ & & $\overline{\mathrm{Ye}}$ & 25 & $\mathrm{~N}$ & & $\mathrm{~N}$ & 0 & No & $\mathrm{N}$ & 0 \\
\hline 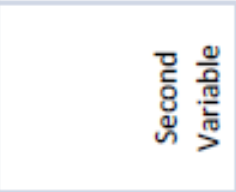 & & & 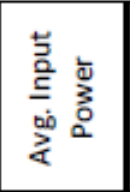 & $\frac{5}{\pi}$ & & 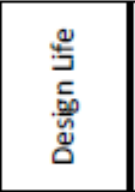 & 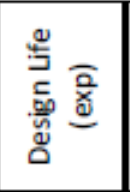 & 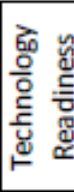 & 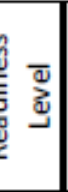 & a & $\frac{2}{5}$ & 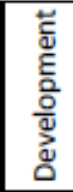 & $\frac{0}{\frac{0}{0}}$ & 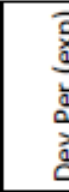 & $\frac{2}{5}$ & 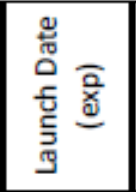 & 농 & \\
\hline Diameter & & & \begin{tabular}{|l|l|}
1.41 & 0.00 \\
\end{tabular} & 1.40 & 0.00 & \begin{tabular}{|l|l|}
1.21 & 0.00 \\
\end{tabular} & \begin{tabular}{|l|l|}
1.13 & 0.00 \\
\end{tabular} & 1.31 & 0.00 & 1.27 & 0.00 & 1.19 & 0.00 & 1.20 & 0.00 & \begin{tabular}{|l|l|}
1.34 & 0.00 \\
\end{tabular} & 1.23 & 0.00 \\
\hline Second Variable & & & \begin{tabular}{|l|l|}
-0.15 & 0.23 \\
\end{tabular} & -0.08 & 0.28 & \begin{tabular}{|l|l|}
-0.01 & 0.98 \\
\end{tabular} & \begin{tabular}{|l|l|}
0.00 & 0.51 \\
\end{tabular} & -0.09 & 0.02 & -0.04 & 0.00 & 0.23 & 0.60 & 0.00 & 0.73 & \begin{tabular}{|l|l|}
-0.04 & 0.00 \\
\end{tabular} & 0.02 & 0.62 \\
\hline Adjusted $\mathrm{r} 2$ & & & $70 \%$ & 91 & $\%$ & \begin{tabular}{|l|}
$71 \%$ \\
\end{tabular} & \begin{tabular}{|l|}
$84 \%$ \\
\end{tabular} & 97 & & 95 & $\%$ & 71 & $\%$ & 71 & $\%$ & $93 \%$ & 66 & $\%$ \\
\hline SPE & & & $58 \%$ & 59 & $\%$ & $83 \%$ & $81 \%$ & 83 & & 39 & $\%$ & 77 & $\%$ & 78 & $\%$ & $39 \%$ & 85 & $\%$ \\
\hline $\mathrm{n}$ & & & 16 & 1. & & 17 & 17 & 8 & & 1 & 6 & 16 & 6 & 1 & 6 & 17 & 1 & 5 \\
\hline Multicollinearity? & & & No & $\mathrm{N}$ & 0 & No & No & $\mathrm{No}$ & & $\mathrm{N}$ & 0 & $\mathrm{Nc}$ & 0 & $\mathrm{~N}$ & 0 & No & $\mathrm{N}$ & 0 \\
\hline
\end{tabular}

Figure 8: Two Variable Model Regression for Optical Telescope Assembly (OTA) Cost vs Aperture Diameter and a $2^{\text {nd }}$ Variable

As with the single variable model, there is utility in looking at OTA Areal Cost. And, the two variable regression confirms the single variable result. It costs less per square meter to build a large aperture space telescope than a small aperture telescope. The regression for OTA Areal Cost is very similar to the regression for OTA Cost, therefore, we will not show a summary chart similar to Figure 8. TRL, YoD and LYr are all significant with a confidence of $>98 \%$. TRL is less noisy, but YoD and LYr have higher correlation values.

$$
\begin{array}{lc}
\text { OTA Areal Cost } \sim \mathrm{D}^{-0.61} \mathrm{e}^{-0.04(\mathrm{LYr}-1960))} & \left(N=17, r_{a d j}^{2}=76 \% ; S P E=40 \%\right) \\
\text { OTA Areal Cost } \sim \mathrm{D}^{-0.68} \mathrm{e}^{-0.04(\mathrm{YoD}-1960))} & \left(N=16, r_{a d j}^{2}=76 \% ; S P E=39 \%\right) \\
\text { OTA Areal Cost } \sim \mathrm{D}^{-0.69} \mathrm{TRL}^{-0.93} & \left(N=8, r_{a d j}^{2}=56 \% ; S P E=35 \%\right)
\end{array}
$$

Like OTA Cost, OTA Areal Cost also reduces as a function of time. It is intuitively obvious that a higher TRL value lowers cost. And the model result should help justify maximizing technology re-use and pre-Phase A development funding. But, given the requirement that all technology should be at TRL-6 before beginning Phase C/D, a TRL based cost model is probably not very useful. Finally, both Mass parameters had terrible $\mathrm{r}_{\text {adj }}^{2}$ values of only $15 \%$.

Next, we look at two variable models for Total Mission Cost. First we used Diameter as the primary variable. Based on a plot of Total Cost versus Diameter (Figure 9), it appears that there is a dependency. And, in fact there may be. However, while the regression yields a coefficient with $99 \%$ significance, its SPE is very noisy:

$$
\text { Total Cost } \sim D^{0.88} \quad\left(N=16, r_{a d j}^{2}=72 \% ; S P E=203 \%\right)
$$


As shown in Figure 10, some second parameters are not multicollinear with diameter and have significant coefficients: Wavelength (75\%), Power (91\%), Design Life (91\%), Development Period (100\%) and Orbit (98\%). And, while it makes sense that all of these parameters might drive total mission cost, none of them make a good statistical model with Diameter. Either the $r_{\text {adj }}^{2}$ value is not high enough or the SPE is too high. So, contrary to everyone's intuition, maybe aperture diameter is not a driver of Total Mission Cost. Or, maybe we have not yet found the right combination of parameters to regress. One thought to be considered in the future is regressing Total Cost against OTA Cost as the primary variable or regressing the difference between Total and OTA cost.

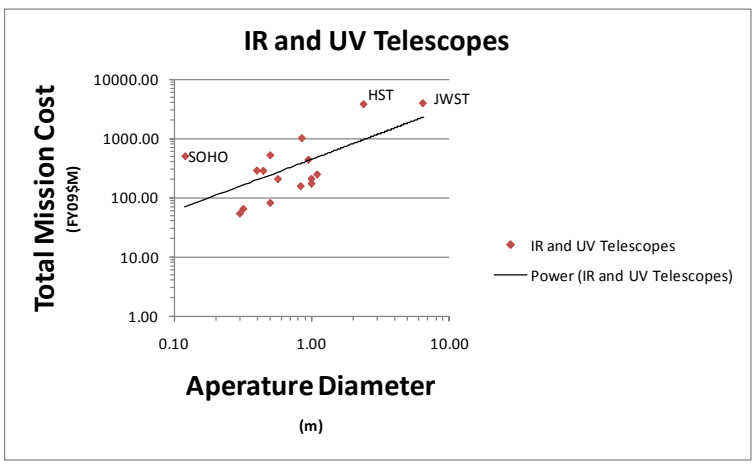

Figure 9: Total Mission Cost vs Aperture Diameter.

\begin{tabular}{|c|c|c|c|c|c|c|c|c|c|c|c|c|c|c|c|c|c|c|c|c|c|c|c|c|}
\hline & \multirow{2}{*}{\multicolumn{2}{|c|}{ 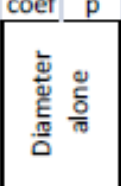 }} & \multicolumn{22}{|c|}{ Total Cost vs Aperture Diameter and V2 } \\
\hline 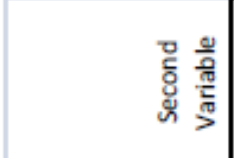 & & & \multicolumn{2}{|c|}{$\begin{array}{l}\frac{\dot{c}}{u} \\
\sum_{a}^{u}\end{array}$} & \multicolumn{2}{|c|}{ 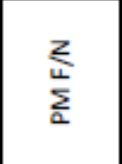 } & \multicolumn{2}{|c|}{ 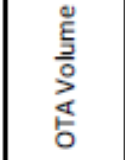 } & \multicolumn{2}{|c|}{ 인 } & \multicolumn{2}{|c|}{ 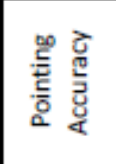 } & \multicolumn{2}{|c|}{ 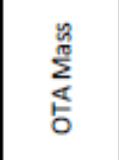 } & \multicolumn{2}{|c|}{ 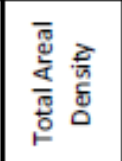 } & \multicolumn{2}{|c|}{$\begin{array}{l}\bar{d} \\
\frac{2}{4} \\
\frac{1}{5} \\
\frac{5}{0}\end{array}$} & \multicolumn{2}{|c|}{ 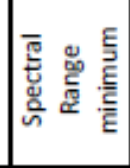 } & \multicolumn{2}{|c|}{ 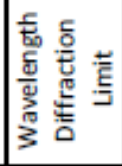 } & \multicolumn{2}{|c|}{ 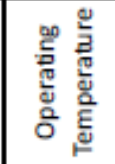 } \\
\hline Aperture Diameter & 0.88 & 0.01 & 0.83 & 0.27 & 1.25 & 0.00 & 0.01 & 1.00 & 0.26 & 0.55 & 0.75 & 0.08 & 1.19 & 0.05 & 2.20 & 0.00 & 1.34 & 0.00 & 0.91 & 0.01 & 0.10 & 0.02 & 0.88 & 0.01 \\
\hline Second Variable & \begin{tabular}{|l|}
- \\
\end{tabular} & \begin{tabular}{|l|}
- \\
\end{tabular} & 0.41 & 0.46 & 0.38 & 0.75 & 0.41 & 0.46 & 0.11 & 0.37 & \begin{tabular}{|l|}
-0.08 \\
\end{tabular} & \begin{tabular}{|l|l|}
0.53 \\
\end{tabular} & 0.08 & 0.83 & 1.03 & 0.00 & 0.08 & 0.83 & -0.08 & 0.55 & -0.23 & 0.25 & -0.04 & 0.81 \\
\hline Adjusted $r^{2}$ & \multicolumn{2}{|c|}{$72 \%$} & \multicolumn{2}{|c|}{\begin{tabular}{|l|}
$61 \%$ \\
\end{tabular}} & \multicolumn{2}{|c|}{$61 \%$} & \multicolumn{2}{|c|}{$61 \%$} & \multicolumn{2}{|c|}{$-11 \%$} & \multicolumn{2}{|c|}{\begin{tabular}{|l|}
$74 \%$ \\
\end{tabular}} & \multicolumn{2}{|c|}{$67 \%$} & \multicolumn{2}{|c|}{$90 \%$} & \multicolumn{2}{|c|}{$67 \%$} & \multicolumn{2}{|c|}{$74 \%$} & \multicolumn{2}{|c|}{$16 \%$} & \multicolumn{2}{|c|}{$68 \%$} \\
\hline SPE & \multicolumn{2}{|c|}{$203 \%$} & \multicolumn{2}{|c|}{$118 \%$} & \multicolumn{2}{|c|}{$119 \%$} & \multicolumn{2}{|c|}{$118 \%$} & 126 & & 178 & $8 \%$ & 115 & $.5 \%$ & 71 & $1 \%$ & 11 & $.5 \%$ & & $76 \%$ & 24 & $1 \%$ & 21 & $9 \%$ \\
\hline n & 1 & 6 & & 12 & 1 & 2 & 12 & 2 & 1 & & 15 & 15 & & 15 & 1 & 15 & 1 & 15 & & 16 & 1 & 10 & 1 & 6 \\
\hline Multicollinearity? & $\mathrm{N}$ & 10 & & es & $\mathrm{N}$ & No & $\mathrm{Ye}$ & es & $\mathrm{N}$ & & $\mathrm{No}$ & Jo & & es & $\mathrm{Ye}$ & es & & No & & No & & Vo & $\mathrm{N}$ & Jo \\
\hline 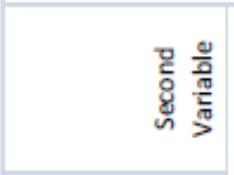 & & & 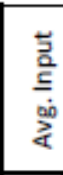 & בे̀ & $\frac{8}{\pi}$ & 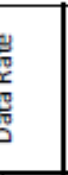 & 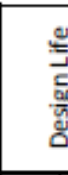 & है & 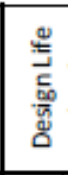 & $\widehat{\underline{a}}$ & 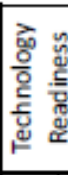 & 怨 & & $\frac{2}{\frac{2}{2}}$ & 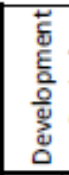 & 은 & है & $\frac{a}{a}$ & 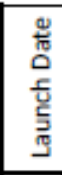 & $\widehat{\frac{a}{x}}$ & & ڤั & & \\
\hline Aperture Diameter & & & 0.31 & 0.44 & 0.55 & 0.26 & 0.54 & 0.13 & 0.54 & 0.11 & 1.77 & 0.01 & 0.95 & 0.01 & 0.52 & 0.05 & 0.36 & 0.16 & 0.94 & 0.01 & 0.86 & 0.00 & & \\
\hline Second Variable & & & 0.37 & 0.09 & 0.14 & 0.36 & 0.56 & 0.11 & 0.01 & 0.09 & -0.52 & 0.45 & -0.03 & 0.35 & 2.01 & 0.00 & 0.03 & 0.00 & -0.02 & 0.60 & 0.16 & 0.02 & & \\
\hline Adjusted $r^{2}$ & & & & $2 \%$ & 22 & $2 \%$ & 84 & $1 \%$ & 66 & $6 \%$ & 93 & $3 \%$ & & $0 \%$ & 77 & $7 \%$ & & $1 \%$ & & $1 \%$ & & $6 \%$ & & \\
\hline SPE & & & & $24 \%$ & 219 & $9 \%$ & 138 & $8 \%$ & 155 & $5 \%$ & 112 & $2 \%$ & 197 & $7 \%$ & 97 & $7 \%$ & & $6 \%$ & 20 & $9 \%$ & & $74 \%$ & & \\
\hline n & & & & 15 & 1 & 2 & 16 & & 1 & 6 & 8 & 8 & & 15 & 15 & 5 & & 15 & & 16 & & 14 & & \\
\hline Multicollinearity? & & & $\mathrm{N}$ & No & $\mathrm{N}$ & Jo & No & & $\mathrm{N}$ & No & $\mathrm{No}$ & Jo & & No & $\mathrm{N}$ & & & No & $\mathrm{N}$ & No & & No & & \\
\hline
\end{tabular}

Figure 10: Two Variable Model Regression for Total Mission Cost vs Aperture Diameter and a $2^{\text {nd }}$ Variable

Now for a disclaimer, we continue to believe that Mass is not an appropriate cost driver (consider that JWST cost more than HST but has half the mass, and that attached OTAs with 10X more mass are 60\% lower cost), but we decided to examine it anyway. Figure 11 shows the two variable regression of Total Mission Cost as a function of Total Mass and a $2^{\text {nd }}$ Variable. Potential models from this regression include:

$$
\begin{aligned}
& \text { Total Cost } \sim \mathrm{TM}^{0.77} \mathrm{e}^{0.02 D e v P e r i o d} \\
& \text { Total Cost } \sim \mathrm{TM}^{0.85} \text { DevPeriod }^{1.16} \\
& \text { Total Cost } \sim \mathrm{TM}^{1.01} \text { Orbit }^{0.09} \\
& \text { Total Cost } \sim \mathrm{TM}^{0.98} \text { DataRate }^{0.10} \\
& \text { Total Cost } \sim \mathrm{TM}^{1.13} \text { Temp }^{-0.13}
\end{aligned}
$$

$$
\begin{aligned}
& \left(N=15, r_{a d j}^{2}=98 \% ; S P E=58 \%\right) \\
& \left(N=15, r_{a d j}^{2}=95 \% ; S P E=54 \%\right) \\
& \left(N=14, r_{a d j}^{2}=96 \% ; S P E=38 \%\right) \\
& \left(N=12, r_{a d j}^{2}=95 \% ; S P E=63 \%\right) \\
& \left(N=15, r_{a d j}^{2}=91 \% ; S P E=56 \%\right)
\end{aligned}
$$

Development Period and Orbit are both 99\% significant. TRL, Operating Temperature and Data Rate are all significant at $>80 \%$ confidence level. But the TRL 'sign' is wrong. Minimum Spectral Wavelength is boarder line significant. One problem with minimum spectral wavelength is that in some cases its coefficient is positive and in other cases it is negative. Neither YoD or LYr are significant. 


\begin{tabular}{|c|c|c|c|c|c|c|c|c|c|c|c|c|c|c|c|c|c|c|c|c|c|c|c|c|}
\hline & \multirow{2}{*}{\multicolumn{2}{|c|}{ 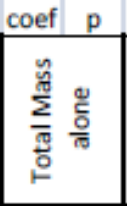 }} & \multicolumn{22}{|c|}{ Total Cost vs Total Mass and V2 } \\
\hline 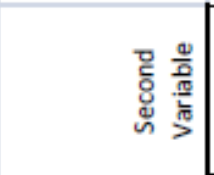 & & & \multicolumn{2}{|c|}{$\begin{array}{l}\stackrel{\dot{\Phi}}{u} \\
\sum \\
\Sigma\end{array}$} & \multicolumn{2}{|c|}{$\frac{\sum}{z}$} & \multicolumn{2}{|c|}{$\begin{array}{l}\text { है } \\
\frac{3}{0} \\
\frac{5}{0} \\
\frac{5}{0}\end{array}$} & \multicolumn{2}{|c|}{ 은 } & \multicolumn{2}{|c|}{ 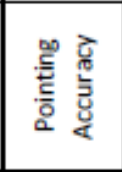 } & \multicolumn{2}{|c|}{ 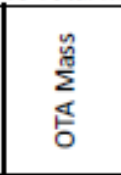 } & \multicolumn{2}{|c|}{ 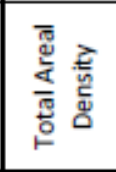 } & \multicolumn{2}{|c|}{$\begin{array}{l}\bar{d} \\
\frac{7}{4} \\
\frac{\mathbb{4}}{4} \\
\frac{5}{0}\end{array}$} & \multicolumn{2}{|c|}{ 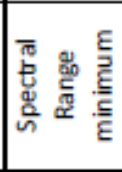 } & \multicolumn{2}{|c|}{ 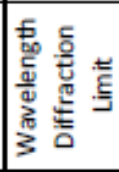 } & \multicolumn{2}{|c|}{ 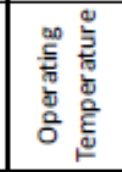 } \\
\hline Total Mass & 1.11 & 0.00 & 1.07 & 0.02 & 1.12 & 0.00 & 0.97 & 0.03 & 1.07 & 0.00 & 1.06 & $0 . \infty$ & 1.20 & 0.00 & 1.10 & 0.00 & 1.06 & 0.00 & 1.14 & 0.00 & 1.03 & 0.00 & 1.13 & 0.00 \\
\hline Second Variable & - & - & 0.03 & 0.93 & -0.14 & 0.75 & 0.06 & 0.71 & 0.04 & 0.61 & -0.03 & 0.60 & -0.08 & 0.69 & -0.06 & 0.54 & $-0.16 \mid$ & 0.47 & 0.09 & 0.20 & $\mid-1.03$ & 0.85 & $\mid-0.13$ & 0.16 \\
\hline Adjusted $r^{2}$ & \multicolumn{2}{|c|}{$85 \%$} & \multicolumn{2}{|c|}{$84 \%$} & \multicolumn{2}{|c|}{\begin{tabular}{|l|}
$81 \%$ \\
\end{tabular}} & \multicolumn{2}{|c|}{$91 \%$} & \multicolumn{2}{|c|}{$9 \%$} & \multicolumn{2}{|c|}{$85 \%$} & \multicolumn{2}{|c|}{$82 \%$} & \multicolumn{2}{|c|}{$90 \%$} & \multicolumn{2}{|c|}{$94 \%$} & \multicolumn{2}{|c|}{$90 \%$} & \multicolumn{2}{|c|}{$26 \%$} & \multicolumn{2}{|c|}{$91 \%$} \\
\hline SPE & \multicolumn{2}{|c|}{$71 \%$} & \multicolumn{2}{|c|}{$81 \%$} & \multicolumn{2}{|c|}{$77 \%$} & \multicolumn{2}{|c|}{$81 \%$} & 79 & $9 \%$ & 72 & $2 \%$ & 81 & $1 \%$ & 71 & $1 \%$ & 87 & $\%$ & & $1 \%$ & & $13 \%$ & & $6 \%$ \\
\hline$n$ & & 5 & & 11 & & 11 & 1 & 1 & 1 & 2 & 1 & 5 & 1 & & 1 & 5 & 1 & 4 & & 15 & & 9 & & 15 \\
\hline Multicollinearity? & & o & & es & & No & $\mathrm{Ye}_{\mathrm{C}}$ & es & $\mathrm{N}$ & lo & $\mathrm{N}$ & lo & $\mathrm{Ye}_{\mathrm{e}}$ & & $\mathrm{N}$ & Jo & $\mathrm{N}$ & lo & & No & & No & & No \\
\hline 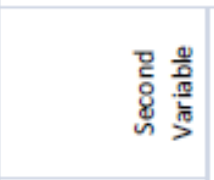 & & & 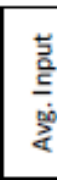 & 产 & s & 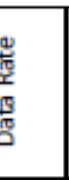 & & & 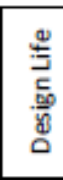 & $\widehat{\widehat{a}}$ & 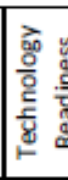 & 这 & क्ष & $\frac{2}{2}$ & 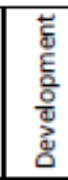 & $\begin{array}{l}\text { 몽 } \\
\text { 음 } \\
\text { ․ }\end{array}$ & a & $\frac{5}{\frac{2}{2}}$ & & $\begin{array}{l}\widehat{a} \\
\frac{d}{5} \\
\frac{5}{5} \\
\frac{1}{3}\end{array}$ & & ڤั & & \\
\hline Total Mass & & & 1.06 & 0.00 & 0.98 & 0.00 & 1.04 & 0.00 & 1.12 & 0.00 & 1.28 & $0 . \infty$ & 1.12 & 0.00 & 0.85 & 0.00 & 0.77 & 0.00 & 1.10 & 0.00 & 1.01 & 0.00 & & \\
\hline Second Variable & & & -0.02 & 0.92 & 0.10 & 0.17 & 0.12 & 0.62 & 0.00 & 0.96 & 0.70 & 0.19 & 0.01 & 0.62 & 1.16 & 0.01 & 0.02 & 0.01 & 0.01 & 0.41 & 0.09 & 0.01 & & \\
\hline Adjusted $r^{2}$ & & & & $4 \%$ & & $5 \%$ & 81 & $1 \%$ & 85 & $5 \%$ & 96 & $5 \%$ & 90 & $\%$ & 95 & $5 \%$ & 98 & $3 \%$ & & $3 \%$ & & $6 \%$ & & \\
\hline SPE & & & & $9 \%$ & & $3 \%$ & 73 & $3 \%$ & 74 & $4 \%$ & 58 & $3 \%$ & 72 & $2 \%$ & 54 & $4 \%$ & 58 & $3 \%$ & & $1 \%$ & & $8 \%$ & & \\
\hline $\mathrm{n}$ & & & & 14 & & 12 & 1 & 5 & 1 & 5 & 8 & 8 & 1. & & 1 & 5 & 1 & 5 & & 15 & & 14 & & \\
\hline Multicollinearity? & & & & No & $\mathrm{N}$ & No & $\mathrm{N}$ & No & $\mathrm{N}$ & No & $\mathrm{N}$ & 10 & $\mathrm{~N}$ & 0 & $\mathrm{~N}$ & Jo & $\mathrm{N}$ & 10 & & No & & No & & \\
\hline
\end{tabular}

Figure 11: Two Variable Model Regression for Total Mission Cost vs Total Mass and a $2^{\text {nd }}$ Variable

\subsection{Three-Variable Models}

The next step after developing a two variable model is to try adding a third parameter. Given that the only satisfactory two variable models were OTA Cost versus Diameter and a 'year' parameter, we did two regressions: Diameter and Year of Development, and Diameter and Launch Year with all the other variables. Both regressions gave similar results with the Year of Launch version yielding slightly better results (Figure 12). None of the regressions yielded a satisfactory model. The only $3^{\text {rd }}$ variable with a significant coefficient was TRL. And, the addition of TRL forced the Launch Year (and Year of Development) coefficient to zero. The only other parameter with an even remotely significant coefficient is Orbit. We will revisit Orbit in the future.

Finally, given the failure of the three variable regression, we decided to add some wavelength diversity by including missions with shorter and longer wavelengths. Specifically, we added WMAP, TDRS-1, TDRS-7, EUVE, Chandra and Einstein. The regression yielded to satisfactory models with the Year of Development being slightly better (Figure 13):

$$
\begin{array}{ll}
\text { OTA Cost } \sim D^{1.15} \lambda^{-0.17} \mathrm{e}^{-0.03(Y o D-1960))} & \left(N=20, r_{a d j}^{2}=92 \% ; S P E=76 \%\right) \\
\text { OTA Cost } \sim D^{1.05} \lambda^{-0.13} \mathrm{e}^{-0.03(L Y-1960))} & \left(N=23, r_{a d j}^{2}=63 \% ; S P E=69 \%\right)
\end{array}
$$

Interestingly, adding wavelength diversity to the regression yields a wavelength coefficients similar to the Horak model:

$$
T 1 \sim A p r^{0.705} \# \text { Elem }{ }^{0.473} \lambda^{-0.178} K^{0-0.191} e^{-0.033(r-80)}
$$

Please note, as indicated by the title of this paper, these results are preliminary and may change over the coming year as we continue our analysis. 


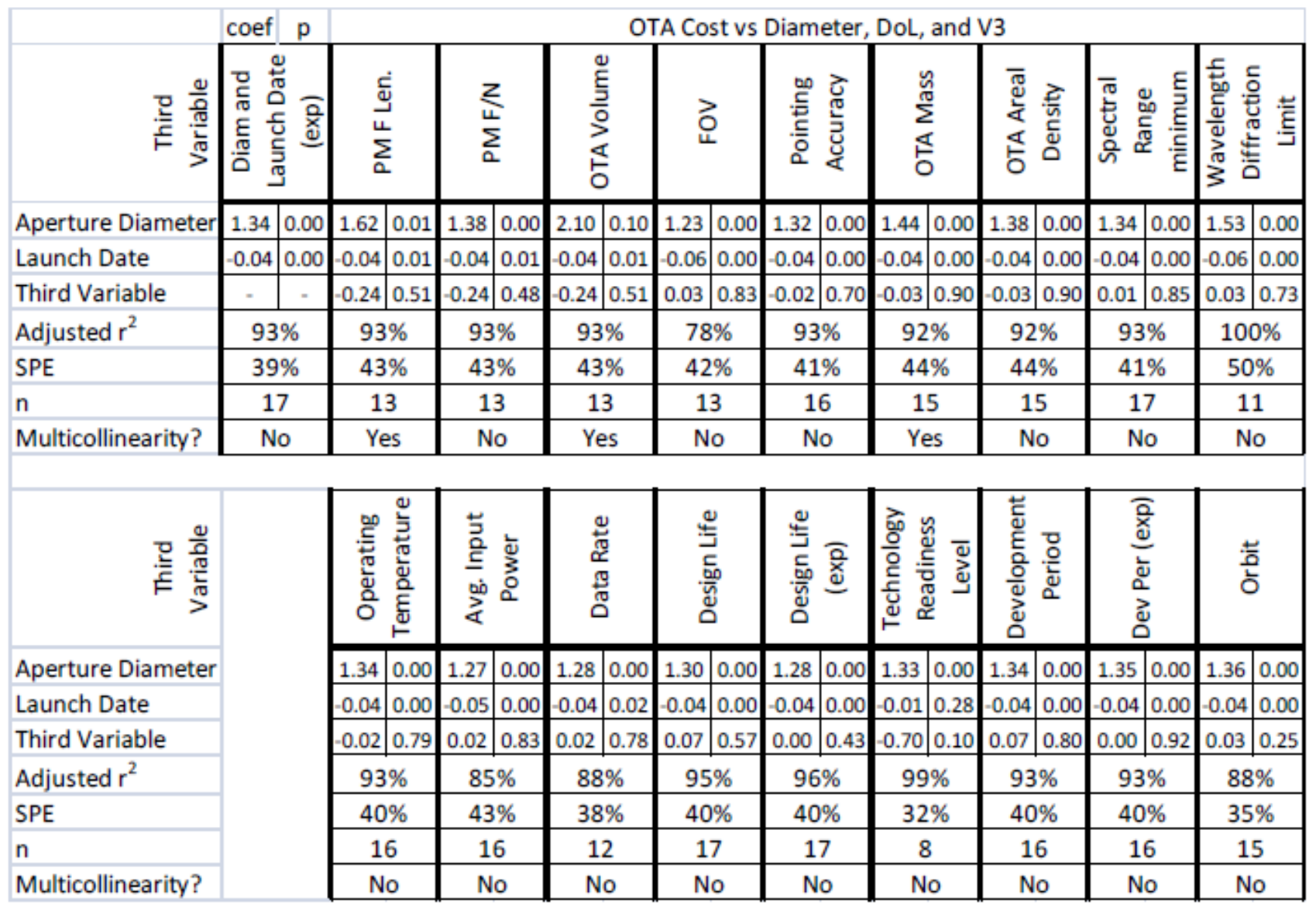

Figure 12: Three Variable Model Regression for OTA Cost vs Diameter, Launch Year and $3^{\text {rd }}$ Parameter

\begin{tabular}{|c|c|c|c|c|c|c|c|c|c|c|c|c|}
\hline \multirow[b]{3}{*}{ Aperture Diameter } & \multirow{2}{*}{\multicolumn{2}{|c|}{$\begin{array}{c}\text { coef } \\
\text { p p } \\
\frac{E}{\frac{\pi}{0}}\end{array}$}} & \multicolumn{10}{|c|}{ OTA Cost vs Diameter, YoD, DoL, and Spct min } \\
\hline & & & \multicolumn{2}{|c|}{ 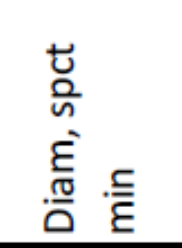 } & \multicolumn{2}{|c|}{$\begin{array}{l}\frac{\widehat{a}}{x} \\
\frac{\varepsilon}{0} \\
\frac{\pi}{0} \\
\frac{0}{\partial}\end{array}$} & \multicolumn{2}{|c|}{ 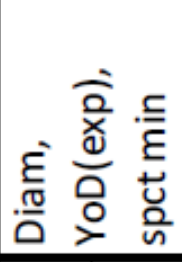 } & \multicolumn{2}{|c|}{ 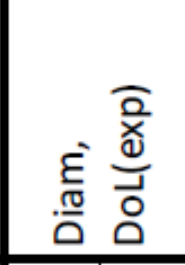 } & \multicolumn{2}{|c|}{ 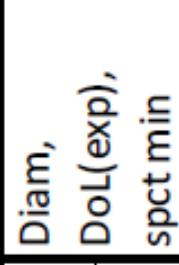 } \\
\hline & 0.84 & 0.00 & 1.03 & 0.00 & 0.78 & 0.00 & 1.15 & 0.00 & 0.85 & 0.00 & 1.05 & 0.00 \\
\hline YoD & - & - & - & - & -0.03 & 0.12 & -0.03 & 0.04 & - & - & - & - \\
\hline Launch Date & - & - & - & - & - & - & - & - & -0.03 & 0.08 & -0.03 & 0.03 \\
\hline Spct Min & - & - & -0.13 & 0.00 & - & - & -0.17 & 0.00 & - & - & -0.13 & 0.00 \\
\hline Adjusted r2 & \multicolumn{2}{|c|}{$43 \%$} & \multicolumn{2}{|c|}{$69 \%$} & \multicolumn{2}{|c|}{$18 \%$} & \multicolumn{2}{|c|}{$92 \%$} & \multicolumn{2}{|c|}{$18 \%$} & \multicolumn{2}{|c|}{$63 \%$} \\
\hline SPE & \multicolumn{2}{|c|}{$126 \%$} & \multicolumn{2}{|c|}{$88 \%$} & \multicolumn{2}{|c|}{$97 \%$} & \multicolumn{2}{|c|}{$76 \%$} & \multicolumn{2}{|c|}{$99 \%$} & \multicolumn{2}{|c|}{$69 \%$} \\
\hline $\mathrm{n}$ & \multicolumn{2}{|c|}{23} & \multicolumn{2}{|c|}{23} & \multicolumn{2}{|c|}{20} & \multicolumn{2}{|c|}{20} & \multicolumn{2}{|c|}{23} & \multicolumn{2}{|c|}{23} \\
\hline Multicollinearity? & \multicolumn{2}{|c|}{$\mathrm{N} / \mathrm{A}$} & \multicolumn{2}{|c|}{ No } & \multicolumn{2}{|c|}{ No } & \multicolumn{2}{|c|}{ No } & \multicolumn{2}{|c|}{ No } & \multicolumn{2}{|c|}{ No } \\
\hline
\end{tabular}

Figure 13: Three Variable Model Regression for OTA Cost vs Diameter, 'year' and Spectral Minimum Wavelength 


\section{CONCLUSIONS}

Cost models are invaluable for system designers. They identify major architectural cost drivers and allow high-level design trades. They enable cost-benefit analysis for technology development investment. And, they provide a basis for estimating total project cost. A study has begun to develop a multivariable parametric cost model for space telescopes. Cost and engineering parametric data has been collected on 30 different missions and extensively analyzed for 23 normal incidence UV/OIR space telescopes. Statistical correlations have been developed for 19 of the 59 variables sampled.

From an engineering \& science perspective, Aperture Diameter is the best parameter for a space telescope cost model. But, the single variable model only predicts $75 \%$ of OTA Cost:

$$
\text { OTA Cost } \sim D^{1.2}\left(N=17 ; r^{2}{ }_{\text {adj }}=75 \% ; \text { SPE }=79 \%\right) \text { with } 2009 \text { JWST }
$$

Two and three variable models provide better estimates:

$$
\begin{array}{ll}
\text { OTA Cost } \sim D^{1.3} \mathrm{e}^{-0.04(\mathrm{LYr}-1960))} & \left(N=17, r_{a d j}^{2}=93 \% ; S P E=39 \%\right) \\
\text { OTA Cost } \sim \mathrm{D}^{1.3} \mathrm{e}^{-0.04(\mathrm{YOD}-1960))} & \left(N=16, r_{a d j}^{2}=95 \% ; S P E=39 \%\right) \\
\text { OTA Cost } \sim \mathrm{D}^{1.15} \lambda^{-0.17} \mathrm{e}^{-0.03(\mathrm{YoD}-1960))} & \left(N=20, r_{a d j}^{2}=92 \% ; S P E=76 \%\right)
\end{array}
$$

where: $\mathrm{D}=$ Aperture Diameter, $\mathrm{LYr}=$ Launch Year, YoD $=$ Year of Development, and $\lambda=$ Spectral Min Wavelength. Similar results were obtained for OTA Areal Cost:

$$
\begin{aligned}
& \text { OTA Areal Cost } \sim \mathrm{D}^{-0.6} \mathrm{e}^{-0.04(\mathrm{LYr}-1960))} \quad\left(N=17, r_{a d j}^{2}=76 \% ; S P E=40 \%\right) \\
& \text { OTA Areal Cost } \sim \mathrm{D}^{-0.7} \mathrm{e}^{-0.04(\mathrm{YoD}-1960))} \quad\left(N=16, r^{2}{ }_{a d j}=76 \% ; S P E=39 \%\right)
\end{aligned}
$$

At present, no satisfactory model has been developed for Total Mission Cost. While total mass does yield a statistically significant result, it contradicts other findings, i.e. that JWST cost more than HST but has half the mass, and that attached OTAs with 10X more mass are $60 \%$ lower cost.

The primary conclusions of the cost modeling study to date are:

- The primary cost driver for Space Telescope Assemblies is Aperture Diameter.

- It costs less per collecting area to build a large aperture telescope than a small aperture telescope.

- Technology development as a function of time reduces cost at the rate of 50\% per 17 years.

- If all other parameters are held constant, adding mass reduces cost.

\section{REFERENCES}

[1] Stahl, H. Philip, "Survey of Cost Models for Space Telescopes", Optical Engineering, Vol.49, No.05, 2010

[2] Stahl, H. Philip, Kyle Stephens, Todd Henrichs, Christian Smart, and Frank A. Prince, "Single Variable Parametric Cost Models for Space Telescopes", Optical Engineering Vol.49, No.06, 2010 